\title{
Global well-posedness of the 3D incompressible porous media equation with critical dissipation in Triebel-Lizorkin spaces
}

\section{Xing Wu, Yanghai Yu and Yanbin Tang* (i)}

\section{${ }^{*}$ Correspondence:}

tangyb@hust.edu.cn

School of Mathematics and

Statistics, Huazhong University of

Science and Technology, Wuhan,

Hubei 430074, P.R. China

\begin{abstract}
In this paper, we study the global well-posedness of the 3D incompressible critical dissipative porous media equation with small initial data in the Triebel-Lizorkin space $F_{p, q}^{s}\left(\mathbb{R}^{3}\right)$. By a pointwise exponential decay estimate on the Poisson semigroup $e^{-t \nu \sqrt{-\Delta}}$ and the Fourier localization technique, we generalize the global well-posedness in the Sobolev spaces $H_{p}^{s}\left(\mathbb{R}^{3}\right)=F_{p, 2}^{s}\left(\mathbb{R}^{3}\right)$ and $H^{s}\left(\mathbb{R}^{3}\right)=F_{2,2}^{s}\left(\mathbb{R}^{3}\right)$ into the general Triebel-Lizorkin spaces $F_{p, q}^{s}\left(\mathbb{R}^{3}\right)$ with $s>\frac{3}{p^{\prime}}, p, q \in(1, \infty)$.
\end{abstract}

MSC: 35Q35; 76D03; 76505

Keywords: porous media equation; global well-posedness; fractional Laplacian; Triebel-Lizorkin spaces

\section{Introduction}

The incompressible flow in porous media bears important significance in mathematical physics [1]. The Cauchy problem of the 3D incompressible dissipative porous media equation assumes the form

$$
\begin{cases}\theta_{t}+u \cdot \nabla \theta+v \Lambda^{\alpha} \theta=0, & x \in \mathbb{R}^{3}, t>0, \\ u(t, x)=-k(\nabla p+g \gamma \theta), & x \in \mathbb{R}^{3}, t>0 \\ \operatorname{div} u(t, x)=0, & x \in \mathbb{R}^{3}, \\ \theta(0, x)=\theta_{0}(x), & \end{cases}
$$

where $0 \leq \alpha \leq 2, \Lambda=\sqrt{-\Delta}$ is the Zygmund operator, $v>0$ is the dissipative coefficient, scalar function $\theta=\theta(t, x)$ is the liquid temperature, $u=u(t, x)$ is the liquid discharge (flux per unit area) to model the flow velocity by the Darcy law, $k$ is the matrix of positionindependent medium permeabilities in the different directions, respectively, divided by the viscosity, $p$ is the pressure, $g$ is the acceleration due to gravity and $\gamma \in \mathbb{R}^{3}$ is the last canonical vector $\mathbf{e}_{3}$. For simplicity, we set $g=1$ and $k=I$, the identity matrix.

The fractional Laplacian $\Lambda^{\alpha}$ is defined through the Fourier transform

$$
\widehat{\Lambda^{\alpha} \theta}(\xi)=|\xi|^{\alpha} \widehat{\theta}(\xi), \quad 0<\alpha<2 .
$$

(c) 2016 Wu et al. This article is distributed under the terms of the Creative Commons Attribution 4.0 International License (http://creativecommons.org/licenses/by/4.0/), which permits unrestricted use, distribution, and reproduction in any medium, provided you give appropriate credit to the original author(s) and the source, provide a link to the Creative Commons license, and indicate if changes were made. 
The cases $0 \leq \alpha<1, \alpha=1,1<\alpha \leq 2$ are called supercritical, critical, subcritical, respectively. Roughly speaking, the critical and supercritical cases are mathematically harder to deal with than the subcritical case. For the fractional Laplacian $\Lambda^{\alpha}$, we refer the reader to [1-4].

According to the Darcy law and the incompressibility condition, for $x \in \mathbb{R}^{3}$ one has [1]

$$
\begin{aligned}
-\Delta_{x} u(t, x) & =\operatorname{curl}(\operatorname{curl} u(t, x)) \\
& =\left(-\frac{\partial^{2} \theta}{\partial x_{1} \partial x_{3}},-\frac{\partial^{2} \theta}{\partial x_{2} \partial x_{3}}, \frac{\partial^{2} \theta}{\partial x_{1}^{2}}+\frac{\partial^{2} \theta}{\partial x_{2}^{2}}\right),
\end{aligned}
$$

using the Newton potential formula and integrating by parts, we have

$$
\begin{aligned}
u(t, x) & =-\frac{2}{3}(0,0, \theta(t, x))+\frac{1}{4 \pi} \text { P.V. } \int_{\mathbb{R}^{3}} K(x-y) \theta(t, y) d y \\
& :=\mathcal{C}(\theta)+\mathcal{S}(\theta),
\end{aligned}
$$

where the integral kernel is

$$
K(x)=\left(\frac{3 x_{1} x_{3}}{|x|^{5}}, \frac{3 x_{2} x_{3}}{|x|^{5}}, \frac{2 x_{3}^{2}-x_{1}^{2}-x_{2}^{2}}{|x|^{5}}\right)
$$

$x=\left(x_{1}, x_{2}, x_{3}\right) \in \mathbb{R}^{3}$, and $\mathcal{C}=\left(\mathcal{C}_{k}\right), \mathcal{S}=\left(\mathcal{S}_{k}\right), 1 \leq k \leq 3$, are all operators mapping scalar functions to vector-valued functions and $\mathcal{C}_{k}$ equals a constant multiplication operator whereas $\mathcal{S}_{k}$ means a Calderón-Zygmund singular integral operator.

The global and local well-posedness of the Cauchy problem (1.1) have been intensively investigated in the last few years.

Córdoba, Gancedo, and Orive [5] studied the analytical behavior of solutions with infinite energy in the case $v=0$ (without dissipation) in the two dimensional space, they obtained the local existence and uniqueness by the particle trajectory method in Hölder spaces $C^{s}\left(\mathbb{R}^{2}\right)$ for $0<s<1$ and gave some blow-up criteria. Very recently, Córdoba, Faraco, and Gancedo [6] proved the non-uniqueness of solutions in $L^{\infty}\left(\mathbb{T}^{2}\right)\left(\mathbb{T}^{2}\right.$ is the two dimensional flat torus) in space and time, Bae and Granero-Belinchón [7] studied transport equations with different nonlocal velocity fields and proved global weak solutions for very rough initial data (merely $L^{1+}$ ) for a one dimensional model of the incompressible porous media equation, and one dimensional and $n$ dimensional models of the dissipative incompressible porous medium equation in the periodic domain.

Castro et al. [1] obtained the existence of strong solutions with regular initial data in the Sobolev space $\theta_{0}(x) \in H^{s}\left(\mathbb{R}^{N}\right)(s>0)$ for the subcritical case $1<\alpha \leq 2$. For the supercritical case $0 \leq \alpha<1$, they also obtained the local well-posedness in the space $H^{s}\left(\mathbb{R}^{N}\right), s>\frac{N-\alpha}{2}+1$, and they extended it to be global under a smallness condition $\left\|\theta_{0}\right\|_{H^{s}}<c v$ on the initial data $\theta_{0} \in H^{s}$ with $s>\frac{N}{2}+1$. In the critical case $\alpha=1$, the existence of strong solutions was obtained. They also proved the global existence of weak solutions with $0 \leq \alpha \leq 2$.

Due to the method established by Hmidi and Keraani [8] for the quasi-geostrophic equation for $0<\alpha<1$, Xue [9] established the local well-posedness of the porous media equation (1.1) in the Besov space $B_{p, 1}^{s}\left(\mathbb{R}^{N}\right)\left(1 \leq p<\infty, s \geq 1+\frac{N}{p}-\alpha\right)$ and in $B_{\infty, 1}^{s}\left(\mathbb{R}^{N}\right) \cap \dot{B}_{\infty, 1}^{0}\left(\mathbb{R}^{N}\right)$ $(s \geq 1-\alpha)$, respectively. Furthermore, Xue [9] also obtained the global well-posedness with small initial data in $\dot{B}_{\infty, 1}^{1-\alpha}\left(\mathbb{R}^{N}\right)$. 
For the critical case $\alpha=1$, by the method of modulus of continuity [10] and the Fourier localization technique, Yuan and Yuan [11] proved the global well-posedness in the critical Besov space $\dot{B}_{p, 1}^{\frac{3}{p}}\left(\mathbb{R}^{3}\right), 1 \leq p \leq \infty$.

Based on Besov space techniques and the method of modulus of continuity, Yamazaki [12] studied the regularized IPM equation in the supercritical regime and the global wellposedness was established in the Sobolev space $H^{m}\left(\mathbb{R}^{3}\right), m \in \mathbb{Z}^{+}, m>\frac{5}{2}$.

We recall that the Triebel-Lizorkin space is a unification of most of the classical function spaces used in partial differential equations such as Lebesgue space $L^{p}\left(\mathbb{R}^{N}\right)$, Sobolev space $H_{p}^{s}\left(\mathbb{R}^{N}\right)$ and Hölder space $C^{s}\left(\mathbb{R}^{N}\right)$ for $s>0$. Chae discussed the local well-posedness and blow-up criterion in the Triebel-Lizorkin space, respectively, for the Euler equation in [13] and for the quasi-geostrophic equation in [14]. Wang and Tang [15-17] studied the long time dynamics of 2D quasi-geostrophic equations.

In this paper, we focus on the critical case $\alpha=1$ in the Triebel-Lizorkin space $F_{p, q}^{s}\left(\mathbb{R}^{3}\right)$ with $s>\frac{3}{p}, p, q \in(1, \infty)$. With the aid of the pointwise exponential decay estimate of the fractional heat semigroup $e^{-t v \Lambda^{\alpha}}$,

$$
\left|e^{-t v \Lambda^{\alpha}} \dot{\Delta}_{j} f(x)\right| \leq C e^{-c t 2^{j \alpha}} \mathcal{M}\left(\dot{\Delta}_{j} f\right)(x), \quad j \in \mathbb{Z}
$$

where $\dot{\Delta}_{j}$ is the Littlewood-Paley projection to the annulus $\left\{|\xi| \sim 2^{j}\right\}$ and $\mathcal{M}$ is the HardyLittlewood maximal operator, if we work in a suitable space-time Triebel-Lizorkin space, after integrating in time we can get $\alpha$ derivatives from (1.2). Especially, for $\alpha=1$, we can obtain the first order derivative which exactly balances the nonlinear term.

Our main result reads as follows.

Theorem 1.1 Assume that $\alpha=1$ and $\theta_{0} \in F_{p, q}^{s}\left(\mathbb{R}^{3}\right)$ with $s>\frac{3}{p}, p, q \in(1, \infty)$. If there exists a positive constant $\epsilon$ such that $\left\|\theta_{0}\right\|_{F_{p, q}^{s}}<\epsilon$, then the Cauchy problem (1.1) of $3 D$ incompressible critical dissipative porous media equation possesses a unique global solution $\theta(t, x)$ such that

$$
\theta(t, x) \in \mathbf{C}\left([0,+\infty) ; F_{p, q}^{s}\right) \cap \tilde{L}^{1}\left(0,+\infty ; \dot{F}_{p, q}^{s+1}\right)
$$

Remark 1.1 Since $F_{p, 2}^{s}\left(\mathbb{R}^{N}\right)=H_{p}^{s}\left(\mathbb{R}^{N}\right)$, Theorem 1.1 implies that the Cauchy problem (1.1) has a global solution with small initial data in the Sobolev space $H_{p}^{s}\left(\mathbb{R}^{3}\right), s>\frac{3}{p}$, $1<p<\infty$.

Throughout this paper, $C$ stands for a constant which may vary from line to line. We shall sometimes use the notation $A \lesssim B$ instead of $A \leq C B$, and $A \approx B$ means that $A \lesssim B$ and $B \lesssim A$.

\section{Preliminaries}

In this section, we provide a characterization of the Triebel-Lizorkin space based on the Littlewood-Paley decomposition. We follow [13, 14, 18-20].

We start with the dyadic partition of unity. Choose two nonnegative radial functions $\chi, \varphi \in \mathcal{S}\left(\mathbb{R}^{N}\right)$, supported, respectively, in the ball $\mathcal{B}=\left\{\xi \in \mathbb{R}^{N},|\xi| \leq \frac{4}{3}\right\}$ and in the ring 
$\mathcal{C}=\left\{\xi \in \mathbb{R}^{N}, \frac{3}{4} \leq|\xi| \leq \frac{8}{3}\right\}$, such that

$$
\begin{aligned}
& \chi(\xi)+\sum_{j \geq 0} \varphi\left(2^{-j} \xi\right)=1, \quad \xi \in \mathbb{R}^{N}, \\
& \sum_{j \in \mathbb{Z}} \varphi\left(2^{-j} \xi\right)=1, \quad \xi \in \mathbb{R}^{N} \backslash\{0\} .
\end{aligned}
$$

Then for $u \in \mathcal{S}^{\prime}\left(\mathbb{R}^{N}\right)$, the homogeneous dyadic block $\dot{\Delta}_{j}$, and the nonhomogeneous dyadic block $\Delta_{j}$ are defined as follows:

$$
\begin{aligned}
& \dot{\Delta}_{j} u(x)=\varphi\left(2^{-j} D\right) u(x)=\mathcal{F}^{-1}\left(\varphi\left(2^{-j} \xi\right) \hat{u}(\xi)\right)(x), \quad \forall j \in \mathbb{Z} ; \\
& \Delta_{j} u(x)=0, \quad j \leq-2 ; \quad \Delta_{-1} u=\chi(D) u, \quad \Delta_{j} u(x)=\varphi\left(2^{-j} D\right) u, \quad j \geq 0 .
\end{aligned}
$$

The homogeneous low-frequency cut-off operator $\dot{S}_{j}$ is defined by

$$
\dot{S}_{j} u=\chi\left(2^{-j} D\right) u, \quad j \in \mathbb{Z} .
$$

It is easily checked that

$$
\begin{aligned}
& \dot{\Delta}_{j} \dot{\Delta}_{k} u=0, \quad|j-k| \geq 2 . \\
& \dot{\Delta}_{j}\left(\dot{S}_{k-1} u \dot{\Delta}_{k} u\right)=0, \quad|j-k| \geq 5 .
\end{aligned}
$$

Using the notations $\dot{\Delta}_{j}$ and $\dot{S}_{j}$, the usual product $u v$ of two distributions $u$ and $v$ can be decomposed into three parts in terms of the paraproduct operators introduced by Bony [19].

Formally, we can write the homogeneous Bony paraproduct decomposition

$$
u v=T_{u} v+T_{v} u+R(u, v)
$$

where

$$
T_{u} v=\sum_{j \in \mathbb{Z}} \dot{S}_{j-1} u \dot{\Delta}_{j} v, \quad R(u, v)=\sum_{j \in \mathbb{Z}} \sum_{|v| \leq 1} \dot{\Delta}_{j-v} u \dot{\Delta}_{j} v
$$

Let us now introduce the Triebel-Lizorkin spaces.

Definition 2.1 Let $s \in \mathbb{R}, p, q \in[1, \infty]$. The homogeneous Triebel-Lizorkin space $\dot{F}_{p, q}^{s}$ is defined by

$$
\dot{F}_{p, q}^{s}\left(\mathbb{R}^{N}\right)=\left\{u \in \mathcal{S}^{\prime}\left(\mathbb{R}^{N}\right) / \mathcal{P}\left(\mathbb{R}^{N}\right),\|u\|_{\dot{F}_{p, q}^{s}}<\infty\right\}
$$

here $\mathcal{S}^{\prime} / \mathcal{P}$ denotes the space of tempered distributions modulus polynomials and

$$
\|u\|_{\dot{F}_{p, q}^{s}}=\|\|\left(2^{j s} \dot{\Delta}_{j} u\right)_{j \in \mathbb{Z}}\left\|_{l^{q}}\right\|_{L^{p}\left(\mathbb{R}^{N}\right)} .
$$


The nonhomogeneous Triebel-Lizorkin space $F_{p, q}^{s}\left(\mathbb{R}^{N}\right)$ is defined by

$$
F_{p, q}^{s}\left(\mathbb{R}^{N}\right)=\left\{u \in \mathcal{S}^{\prime}\left(\mathbb{R}^{N}\right),\|u\|_{F_{p, q}^{s}}<\infty\right\}
$$

with

$$
\|u\|_{F_{p, q}^{s}}\left(\mathbb{R}^{N}\right)=\|\|\left(2^{j s} \Delta_{j} u\right)_{j \geq-1}\left\|_{l^{q}}\right\|_{L^{p}\left(\mathbb{R}^{N}\right)} .
$$

Remark 2.1 We point out that if $s>0$, we have $F_{p, q}^{s}\left(\mathbb{R}^{N}\right)=\dot{F}_{p, q}^{s}\left(\mathbb{R}^{N}\right) \cap L^{p}\left(\mathbb{R}^{N}\right)$, then by the definition of the nonhomogeneous Triebel-Lizorkin space, the Minkowski inequality, and the fact that $\left\|\Delta_{-1} u\right\|_{L^{p}} \leq C\|u\|_{L^{p}}$, we get

$$
\begin{aligned}
\|u\|_{F_{p, q}^{s}} & \approx\|u\|_{\dot{F}_{p, q}^{s}}+\|u\|_{L^{p}} \\
& \approx\|u\|_{\dot{F}_{p, q}^{s}}+\left\|\Delta_{-1} u\right\|_{L^{p}} .
\end{aligned}
$$

Remark 2.2 (Chae [13]) If $s>\frac{N}{p}, F_{p, q}^{s}\left(\mathbb{R}^{N}\right) \hookrightarrow L^{\infty}\left(\mathbb{R}^{N}\right), p, q \in[1, \infty]$.

The following space-time Triebel-Lizorkin space will play an important role in the proof of Theorem 1.1.

Definition 2.2 Let $s \in \mathbb{R}, p, q, r \in[1, \infty], I \subset \mathbb{R}$ be an interval. The homogeneous spacetime Triebel-Lizorkin space $\tilde{L}^{r}\left(I ; \dot{F}_{p, q}^{s}\left(\mathbb{R}^{N}\right)\right)$ is the set of all distributions satisfying

$$
\|u\|_{\tilde{L}^{r}\left(I ; \dot{F}_{p, q}^{s}\right)}=\|\|\left(2^{j s}\left\|\dot{\Delta}_{j} u\right\|_{L_{t}^{r}(I)}\right)_{j \in \mathbb{Z}}\left\|_{l q}\right\|_{L^{p}}<\infty
$$

We can also define the inhomogeneous space-time Triebel-Lizorkin space $\tilde{L}^{r}\left(I ; F_{p, q}^{s}\left(\mathbb{R}^{N}\right)\right)$. By Remark 2.1, if $s>0$,

$$
\begin{aligned}
\|u\|_{\tilde{L}^{r}\left(I ; F_{p, q}^{s}\right)} & \approx\|u\|_{\tilde{L}^{r}\left(I ; \dot{F}_{p, q}^{s}\right)}+\|u\|_{L_{t}^{r}\left(I ; L^{p}\right)} \\
& \approx\|u\|_{\tilde{L}^{r}\left(I ; \dot{F}_{p, q}^{s}\right)}+\left\|\Delta_{-1} u\right\|_{L_{t}^{r}\left(I ; L^{p}\right)}
\end{aligned}
$$

For simplicity, we use $\tilde{L}_{t}^{r} \dot{F}_{p, q}^{s}, \tilde{L}^{r} \dot{F}_{p, q}^{s}$ to denote $\tilde{L}^{r}\left(0, t ; \dot{F}_{p, q}^{s}\right)$ and $\tilde{L}^{r}\left(0, \infty ; \dot{F}_{p, q}^{s}\right)$, respectively. For a locally integrable function $f$, the Hardy-Littlewood maximal function $\mathcal{M} f$ is defined by

$$
\mathcal{M} f(x)=\sup _{r>0} \frac{1}{|\mathcal{B}(x, r)|} \int_{\mathcal{B}(x, r)}|f(y)| d y,
$$

where $|\mathcal{B}(x, r)|$ denotes the volume of the ball $\mathcal{B}(x, r)$ with center $x$ and radius $r$.

The following vector-valued maximal inequality, which can be found in [21], plays a fundamental tool in the proof of product estimate.

Lemma 2.1 (Vector-valued maximal inequality) Let $1<p<\infty, 1 \leq q \leq \infty$, and $\left\{u_{j}\right\}_{j \in \mathbb{Z}}$ be a sequence of functions in $L^{p}\left(l^{q}\right)$. Then we have

$$
\|\| \mathcal{M} u_{j}\left\|_{l^{q}}\right\|_{L^{p}} \lesssim\|\| u_{j}\left\|_{l^{q}}\right\|_{L^{p}}
$$


The following lemma is proved by Stein [22].

Lemma 2.2 Let $\phi$ be an integrable function on $\mathbb{R}^{N}$ and the least decreasing radial majorant of $\phi$ be integrable, i.e.,

$$
\int_{\mathbb{R}^{N}} \sup _{|y| \geq|x|}|\phi(y)| d x=A<\infty
$$

Then for any $u \in L^{p}\left(\mathbb{R}^{N}\right), 1 \leq p \leq \infty$, we have

$$
\sup _{\epsilon>0} \epsilon^{N}|(u * \phi(\epsilon \cdot))(x)| \leq A \mathcal{M} u(x)
$$

Lemma 2.3 (Frazier-Torres-Weiss [23]) The Calderón-Zygmund singular integral operator is bounded from the Triebel-Lizorkin space $F_{p, q}^{s}$ into itself.

Using Lemma 2.3 , we can control $u$ constantly by $\theta$ modulus multiplication by a constant in the space $F_{p, q}^{s}$.

Finally, let us recall the maximum principle.

Lemma 2.4 Let $\theta$ be the smooth solution to the Cauchy problem (1.1) with $\alpha \in(0,2)$. Then we have

$$
\|\theta(t)\|_{L^{p}} \leq\|\theta(0)\|_{L^{p}}, \quad 1 \leq p \leq \infty
$$

Proof Hmidi and Keraani [8] established a maximum principle (2.9) for the quasigeostrophic equation and the result does not depend on the space dimension. Following the idea of proof in [8] we can prove that (2.9) holds also for the 3D incompressible porous media equation similarly. Here we omit it.

Remark 2.3 We can get an explicit decay estimates of the $L^{p}$ norm in $\theta$ using the methods developed by Córdoba and Córdoba [24], however, the boundedness is enough for the proof of Theorem 1.1.

\section{Proof of Theorem 1.1}

In this section, we prove Theorem 1.1. We divide the proof into four steps.

Step 1. A priori estimates. Firstly, we rewrite (1.1) in the following integral form:

$$
\theta(t)=e^{-v t \Lambda} \theta_{0}-\int_{0}^{t} e^{-(t-s) v \Lambda} \nabla \cdot(u \theta)(s) d s
$$

where we have used the fact that $\nabla \cdot u=0$.

Then localizing (3.1) through the Fourier localization operator $\dot{\Delta}_{j}$ and using estimates in Lemma A.2 in the Appendix, we get

$$
\left|\dot{\Delta}_{j} \theta(t, x)\right| \lesssim e^{-c v t 2^{j}} \mathcal{M}\left(\dot{\Delta}_{j} \theta_{0}\right)(x)+\int_{0}^{t} e^{-c v(t-s) 2^{j}} 2^{j} \mathcal{M}\left(\dot{\Delta}_{j}(u \theta)\right)(s, x) d s
$$


Taking $L^{\infty}, L^{1}$ norm, respectively, with respect to $t$ on both sides of (3.2) and using Young's inequality give

$$
\begin{aligned}
\left\|\dot{\Delta}_{j} \theta(\cdot, x)\right\|_{L_{t}^{\infty}} & \lesssim \mathcal{M}\left(\dot{\Delta}_{j} \theta_{0}\right)(x)+2^{j}\left\|\mathcal{M}\left(\dot{\Delta}_{j}(u \theta)\right)(\cdot, x)\right\|_{L_{t}^{1}}, \\
\left\|\dot{\Delta}_{j} \theta(\cdot, x)\right\|_{L_{t}^{1}} & \lesssim \frac{1}{v} 2^{-j} \mathcal{M}\left(\dot{\Delta}_{j} \theta_{0}\right)(x)+\frac{1}{v}\left\|\mathcal{M}\left(\dot{\Delta}_{j}(u \theta)\right)(\cdot, x)\right\|_{L_{t}^{1}}
\end{aligned}
$$

Multiplying $2^{j s}$ on both sides of (3.3), taking $l^{q}(\mathbb{Z})$ norm, then taking $L^{p}$ norm, we get

$$
\|\theta\|_{\tilde{L}^{\infty} \dot{F}_{p, q}^{s}} \lesssim\left\|\theta_{0}\right\|_{\dot{F}_{p, q}^{s}}+\|u \theta\|_{\tilde{L}^{1} \dot{F}_{p, q}^{s+1}}
$$

due to the Lemma 2.1, using the product estimate in Lemma A.1 we obtain

$$
\begin{aligned}
\|\theta\|_{\tilde{L}^{\infty} \dot{F}_{p, q}^{s}} & \lesssim\left\|\theta_{0}\right\|_{\dot{F}_{p, q}^{s}}+\|u\|_{\tilde{L}_{t}^{\infty} L_{x}^{\infty}}\|\theta\|_{\tilde{L}_{t}^{1} \dot{F}_{p, q}^{s+1}}+\|\theta\|_{\tilde{L}_{t}^{\infty} L_{x}^{\infty}}\|u\|_{\tilde{L}_{t}^{1} \dot{F}_{p, q}^{s+1}} \\
& \lesssim\left\|\theta_{0}\right\|_{\dot{F}_{p, q}^{s}}+\|u\|_{\tilde{L}_{t}^{\infty} F_{p, q}^{s}}\|\theta\|_{\tilde{L}_{t}^{1} \dot{F}_{p, q}^{s+1}}+\|\theta\|_{\tilde{L}_{t}^{\infty} F_{p, q}^{s}}\|u\|_{\tilde{L}_{t}^{1} \dot{s}_{p, q}^{s+1}}
\end{aligned}
$$

according to the property of the Calderón-Zygmund singular integral operator in Lemma 2.3 we get

$$
\|\theta\|_{\tilde{L}_{t}^{\infty} \dot{F}_{p, q}^{s}} \lesssim\left\|\theta_{0}\right\|_{\dot{F}_{p, q}^{s}}+\|\theta\|_{\tilde{L}_{t}^{1} \dot{F}_{p, q}^{s+q}}\|\theta\|_{\tilde{L}_{t}^{\infty} F_{p, q}^{s}}
$$

Similarly to (3.5), we get

$$
v\|\theta\|_{\tilde{L}_{t}^{1} \dot{s}_{p, q}^{s+1}} \lesssim\left\|\theta_{0}\right\|_{\dot{F}_{p, q}^{s}}+\|\theta\|_{\tilde{L}_{t}^{1} \dot{F}_{p, q}^{s+1}}\|\theta\|_{\tilde{L}_{t}^{\infty} F_{p, q}^{s}}
$$

Combining (3.5), (3.6), and using Lemma 2.4, we get

$$
\|\theta\|_{\tilde{L}_{t}^{\infty} F_{p, q}^{s}}+v\|\theta\|_{\tilde{L}_{t}^{1} \dot{s}_{p, q}^{s+1}} \lesssim\left\|\theta_{0}\right\|_{F_{p, q}^{s}}+\|\theta\|_{\tilde{L}_{t}^{1} \dot{F}_{p, q}^{s+1}}\|\theta\|_{\tilde{L}_{t}^{\infty} F_{p, q}^{s}}
$$

Step 2. Approximate solutions and uniform estimates. We construct the following successive approximate sequence $\left\{\theta^{n}\right\}$ :

$$
\begin{cases}\partial_{t} \theta^{n+1}+u^{n} \cdot \nabla \theta^{n+1}+v \Lambda \theta^{n+1}=0, & x \in \mathbb{R}^{3}, t>0 \\ u^{n}=\mathcal{C}\left(\theta^{n}\right)+\mathcal{S}\left(\theta^{n}\right), & x \in \mathbb{R}^{3}, t>0 \\ \operatorname{div} u^{n}=0, & \\ \theta^{n+1}(0, x)=S_{n+2} \theta_{0}, & \end{cases}
$$

where $S_{j}(j \geq 0)$ are low-frequency cut-off operators which are defined similarly by $(2.5)$ :

$$
S_{j} u=\chi\left(2^{-j} D\right) u, \quad j \in \mathbb{N} \cup 0 .
$$

Setting $\left(\theta^{0}, u^{0}\right)=(0,0)$, and solving the linear system, we can find $\left\{\theta^{n}, u^{n}\right\}_{n \in \mathbb{N}}$ for all $n \in \mathbb{N}$. As in Step 1 , we can deduce that

$$
\begin{aligned}
& \left\|\theta^{n+1}\right\|_{\tilde{L}_{t}^{\infty} F_{p, q}^{s}}+v\left\|\theta^{n+1}\right\|_{\tilde{L}_{t}^{1} \dot{F}_{p, q}^{s+1}} \\
& \quad \leq C\left(\left\|\theta_{0}^{n+1}\right\|_{F_{p, q}^{s}}+\left\|\theta^{n}\right\|_{\tilde{L}_{t}^{1} \dot{F}_{p, q}^{s+1}}\left\|\theta^{n+1}\right\|_{\tilde{L}_{t}^{\infty} F_{p, q}^{s}}+\left\|\theta^{n}\right\|_{\tilde{L}_{t}^{\infty} F_{p, q}^{s}}\left\|\theta^{n+1}\right\|_{\tilde{L}_{t}^{1} \dot{t}_{p, q}^{s+1}}\right) .
\end{aligned}
$$


If we take $\epsilon>0$, such that $\left\|\theta_{0}\right\|_{F_{p, q}^{s}} \leq \epsilon, \epsilon \leq \frac{v}{4 C^{2}}$, then we claim that, for all $n \in \mathbb{N}$,

$$
\left\|\theta^{n}\right\|_{\tilde{L}^{\infty} F_{p, q}^{s}}+v\left\|\theta^{n}\right\|_{\tilde{L}^{1} \dot{F}_{p, q}^{s+1}} \leq 2 C\left\|\theta_{0}\right\|_{F_{p, q}^{s}} .
$$

In fact, assume that

$$
\left\|\theta^{n}\right\|_{\tilde{L}^{\infty} F_{p, q}^{s}}+\nu\left\|\theta^{n}\right\|_{\tilde{L}_{1} \dot{F}_{p, q}^{s+1}} \leq 2 C\left\|\theta_{0}\right\|_{F_{p, q}^{s}} .
$$

It follows from (3.9) that

$$
\begin{aligned}
& \left\|\theta^{n+1}\right\|_{\tilde{L}_{t}^{\infty} F_{p, q}^{s}}+v\left\|\theta^{n+1}\right\|_{\tilde{L}_{t}^{1} \dot{H}_{p, q}^{s+1}} \\
& \quad \leq C\left\|\theta_{0}\right\|_{F_{p, q}^{s}}+\frac{C}{v}\left(\left\|\theta^{n}\right\|_{\tilde{L}_{t}^{\infty} F_{p, q}^{s}}+v\left\|\theta^{n}\right\|_{\tilde{L}_{t}^{1} \dot{\dot{H}}_{p, q}^{s+1}}\right)\left(\left\|\theta^{n+1}\right\|_{\tilde{L}_{t}^{\infty} F_{p, q}^{s}}+v\left\|\theta^{n+1}\right\|_{\tilde{L}_{t}^{1} \dot{F}_{p, q}^{s+1}}\right) \\
& \quad \leq C\left\|\theta_{0}\right\|_{F_{p, q}^{s}}+\frac{1}{2}\left(\left\|\theta^{n+1}\right\|_{\tilde{L}^{\infty} F_{p, q}^{s}}+v\left\|\theta^{n+1}\right\|_{\tilde{L}^{1} \dot{F}_{p, q}^{s+q}}\right),
\end{aligned}
$$

which implies (3.10).

Step 3. Compactness arguments and existence. We will show that the sequence $\left\{\theta^{n}\right\}$ has a subsequence converging to a solution of the Cauchy problem (1.1) in $\mathcal{D}^{\prime}\left(\mathbb{R}^{+} \times \mathbb{R}^{3}\right)$. The proof is based on compactness arguments.

Firstly, we show that $\partial_{t} \theta^{n}$ is uniformly bounded in space-time Triebel-Lizorkin $L_{t}^{\infty}\left(F_{p, q}^{s-1}\right)$. By (3.8), we have

$$
\begin{aligned}
\left\|\partial_{t} \theta^{n+1}\right\|_{F_{p, q}^{s, 1}} & \leq\left\|\nabla \cdot\left(u^{n} \theta^{n+1}\right)\right\|_{F_{p, q}^{s,-1}}+v\left\|\Lambda \theta^{n+1}\right\|_{F_{p, q}^{s-1}} \\
& \leq\left\|u^{n} \theta^{n+1}\right\|_{F_{p, q}^{s}}+v\left\|\theta^{n+1}\right\|_{F_{p, q}^{s}},
\end{aligned}
$$

due to the fact that $F_{p, q}^{s}\left(s>\frac{3}{p}\right)$ is a Banach algebra and the property of the CalderónZygmund singular integral operator in Lemma 2.3,

$$
\begin{aligned}
\left\|\partial_{t} \theta^{n+1}\right\|_{F_{p, q}^{s-1}} & \leq\left\|u^{n}\right\|_{F_{p, q}^{s}}\left\|\theta^{n+1}\right\|_{F_{p, q}^{s}}+v\left\|\theta^{n+1}\right\|_{F_{p, q}^{s}} \\
& \leq C\left\|\theta^{n}\right\|_{F_{p, q}^{s}}\left\|\theta^{n+1}\right\|_{F_{p, q}^{s}}+v\left\|\theta^{n+1}\right\|_{F_{p, q}^{s}} .
\end{aligned}
$$

On the other hand, since $\tilde{L}^{\infty} F_{p, q}^{s} \hookrightarrow L^{\infty} F_{p, q}^{s},(3.10)$ and (3.11) imply that

$$
\left\|\partial_{t} \theta^{n+1}\right\|_{L^{\infty} F_{p, q}^{s-1}}<\infty
$$

Now let us turn to the proof of the existence. We note that for any $\psi \in \mathcal{S}\left(\mathbb{R}^{3}\right)$, the map $u \longmapsto \psi u$ is compact from $H_{p}^{s}$ into $H_{p}^{t}$ for $s>t, p<\infty$, together with the Sobolev embedding

$$
F_{p, q}^{s} \hookrightarrow F_{p, 2}^{s-\epsilon}=H_{p}^{s-\epsilon} \hookrightarrow L^{p}, \quad \forall s>\epsilon, \epsilon>0
$$

we thus get the map $u \longmapsto \psi u$ is compact from $F_{p, q}^{s}\left(\mathbb{R}^{3}\right)$ into $L^{p}\left(\mathbb{R}^{3}\right)$. Thus by the LionsAubin compactness theorem, we can conclude that there exist a subsequence $\left\{\theta^{n_{k}}\right\}$ and a 
function $\theta$ such that

$$
\lim _{k \rightarrow \infty} \theta^{n_{k}}=\theta \quad \text { in } L_{\mathrm{loc}}^{P}\left(R^{+} \times \mathbb{R}^{3}\right) .
$$

Moreover, the uniform estimate (3.10) allows us to conclude that $\theta(t, x) \in \tilde{L}^{\infty} F_{p, q}^{s} \cap \tilde{L}^{1} \dot{F}_{p, q}^{s+1}$, and

$$
\|\theta\|_{\tilde{L}^{\infty} F_{p, q}^{s}}+\nu\|\theta\|_{\tilde{L}^{1} \dot{F}_{p, q}^{s+1}} \leq 2 C\left\|\theta_{0}\right\|_{F_{p, q}^{s}} \cdot
$$

Finally, by a standard limit argument, we can prove that the limit function $\theta(t, x)$ satisfies the Cauchy problem (1.1) in the sense of distribution.

We still have to prove that $\theta(t, x) \in \mathcal{C}\left(0, \infty ; F_{p, q}^{s}\right)$. From the definition of the TriebelLizorkin space and the Minkowski inequality we have

$$
\begin{aligned}
\left\|\theta(t)-\theta\left(t^{\prime}\right)\right\|_{F_{p, q}^{s}} \leq & \left\|\left(\sum_{j<N} 2^{j s q}\left|\Delta_{j} \theta(t)-\Delta_{j} \theta\left(t^{\prime}\right)\right|^{q}\right)^{\frac{1}{q}}\right\|_{L^{p}} \\
& +2\left\|\left(\sum_{j \geq N} 2^{j s q}\left\|\Delta_{j} \theta\right\|_{L_{t}^{\infty}}^{q}\right)^{\frac{1}{q}}\right\|_{L^{p}}, \quad \forall N \in \mathbb{N} .
\end{aligned}
$$

From (3.13), for $\epsilon>0$, there exists a number $N_{0} \in \mathbb{N}$ such that

$$
\left\|\left(\sum_{j \geq N_{0}} 2^{j s q}\left\|\Delta_{j} \theta\right\|_{L_{t}^{\infty}}^{q}\right)^{\frac{1}{q}}\right\|_{L^{p}} \leq \frac{\epsilon}{4},
$$

while

$$
\begin{aligned}
\left\|\left(\sum_{j<N_{0}} 2^{j s q}\left|\Delta_{j} \theta(t)-\Delta_{j} \theta\left(t^{\prime}\right)\right|^{q}\right)^{\frac{1}{q}}\right\|_{L^{p}} & \lesssim\left|t-t^{\prime}\right| \cdot\left\|\left(\sum_{j<N_{0}} 2^{j s q}\left\|\partial_{t} \Delta_{j} \theta\right\|_{L_{\mathbb{R}^{+}}^{\infty}}^{q}\right)^{\frac{1}{q}}\right\|_{L^{p}} \\
& \lesssim\left|t-t^{\prime}\right| 2^{N_{0}}\left\|\partial_{t} \theta\right\|_{\tilde{L}^{\infty} F_{p, q}^{s-1}} .
\end{aligned}
$$

Equation (3.12) allows us to finish the proof of continuity in time, that is, $\theta(t, x) \in$ $\mathcal{C}\left(0, \infty ; F_{p, q}^{s}\right)$.

Step 4. Uniqueness. Assume that $\theta^{\prime}(t, x) \in \tilde{L}^{\infty} F_{p, q}^{s} \cap \tilde{L}^{1} \dot{F}_{p, q}^{s+1}$ is another solution of the Cauchy problem (1.1) with the same initial data $\theta_{0}(x)$.

Let $\delta \theta=\theta-\theta^{\prime}$ and $\delta u=u-u^{\prime}$, then $(\delta \theta, \delta u)$ satisfies the following Cauchy problem:

$$
\left\{\begin{array}{l}
\partial_{t} \delta \theta+u \cdot \nabla \delta \theta+v \Lambda \delta \theta=-\delta u \cdot \nabla \theta^{\prime}, \quad x \in \mathbb{R}^{3}, t>0 \\
\operatorname{div} u=0 \\
\delta \theta(0, x)=0
\end{array}\right.
$$

Following the same procedure of estimate leading to (3.7), by Lemma A.1 in the Appendix and Lemma 2.3 we can obtain

$$
\begin{aligned}
& \|\delta \theta\|_{\tilde{L}_{T}^{\infty} \dot{F}_{p, q}^{s}}+v\|\delta \theta\|_{\tilde{L}_{T}^{1} \dot{F}_{p, q}^{s+1}} \\
& \quad \lesssim\|u \cdot \delta \theta\|_{\tilde{L}_{T}^{1} \dot{F}_{p, q}^{s+1}}+\left\|\delta u \cdot \theta^{\prime}\right\|_{\tilde{L}_{T}^{1} \dot{F}_{p, q}^{s+1}}
\end{aligned}
$$




$$
\begin{aligned}
\lesssim & \|u\|_{\tilde{L}_{T}^{\infty} F_{p, q}^{s}}\|\delta \theta\|_{\tilde{L}_{T}^{1} \dot{F}_{p, q}^{s+1}}+\|\delta \theta\|_{\tilde{L}_{T}^{\infty} F_{p, q}^{s}}\|u\|_{\tilde{L}_{T}^{1} \dot{F}_{p, q}^{s+1}} \\
& +\|\delta u\|_{\tilde{L}_{T}^{\infty} F_{p, q}^{s}}\left\|\theta^{\prime}\right\|_{\tilde{L}_{T}^{1} \dot{F}_{p, q}^{s+1}}+\left\|\theta^{\prime}\right\|_{\tilde{L}_{T}^{\infty} F_{p, q}^{s}}\|\delta u\|_{\tilde{L}_{T}^{1} \dot{F}_{p, q}^{s+1}} \\
\lesssim & \|\delta \theta\|_{\tilde{L}_{T}^{\infty} F_{p, q}^{s}}\left(\|\theta\|_{\tilde{L}_{T}^{1} \dot{F}_{p, q}^{s+1}}+\left\|\theta^{\prime}\right\|_{\tilde{L}_{T}^{1} \dot{F}_{p, q}^{s+1}}\right) \\
& +\|\delta \theta\|_{\tilde{L}_{T}^{1} \dot{F}_{p, q}^{s+1}}\left(\|\theta\|_{\tilde{L}_{T}^{\infty} F_{p, q}^{s}}+\left\|\theta^{\prime}\right\|_{\tilde{L}_{T}^{\infty} F_{p, q}^{s}}\right) .
\end{aligned}
$$

In order to get the estimates in the inhomogeneous mixed time-space Triebel-Lizorkin space, it remains to estimate $\left\|\Delta_{-1}(\delta \theta)\right\|_{L_{T}^{\infty} L_{x}^{p}}$. To do this, we apply the operator $\Delta_{-1}$ on both sides of (3.14). Modifying slightly the proof of Proposition 6.2 in [8], together with the maximum principle, Bernstein's inequality [19], and Hölder's inequality yield

$$
\begin{aligned}
\left\|\Delta_{-1}(\delta \theta)\right\|_{L_{T}^{\infty} L_{x}^{p}} & \leq \int_{0}^{T}\left\|\Delta_{-1}\left(\delta u \cdot \nabla \theta^{\prime}+u \cdot \nabla \delta \theta\right)\right\|_{L^{p}} d t \\
& \leq \int_{0}^{T}\left\|\nabla \cdot \Delta_{-1}\left(\theta^{\prime} \delta u+u \delta \theta\right)\right\|_{L^{p}} d t \\
& \lesssim \int_{0}^{T}\left\|\theta^{\prime} \delta u+u \delta \theta\right\|_{L^{p}} d t,
\end{aligned}
$$

thus the property of Calderón-Zygmund singular integral operator in Lemma 2.3 and the Sobolev embedding $F_{p, q}^{s} \hookrightarrow L^{p}(s>0)$ imply that

$$
\begin{aligned}
\left\|\Delta_{-1}(\delta \theta)\right\|_{L_{T}^{\infty} L_{x}^{p}} & \lesssim \int_{0}^{T}\left(\|u\|_{L_{x}^{\infty}}\|\delta \theta\|_{L^{p}}+\|\delta u\|_{L^{p}}\left\|\theta^{\prime}\right\|_{L_{x}^{\infty}}\right) d t \\
& \lesssim T\left(\|\theta\|_{\tilde{L}_{T}^{\infty} F_{p, q}^{s}}+\left\|\theta^{\prime}\right\|_{\tilde{L}_{T}^{\infty} F_{p, q}^{s}}\right)\|\delta \theta\|_{\tilde{L}_{T}^{\infty} F_{p, q}^{s}}
\end{aligned}
$$

Combining (3.15) and (3.16), we get

$$
\begin{aligned}
\|\delta \theta\|_{\tilde{L}_{T}^{\infty} F_{p, q}^{s}}+\|\delta \theta\|_{\tilde{L}_{T}^{1} \dot{F}_{p, q}^{s+1}} \leq & C\left(\|\delta \theta\|_{\tilde{L}_{T}^{\infty} F_{p, q}^{s}}+\|\delta \theta\|_{\tilde{L}_{T}^{1} \dot{F}_{p, q}^{s+q}}\right) \\
& \cdot\left(\|\theta\|_{\tilde{L}_{T}^{1} \dot{F}_{p, q}^{s+1}}+(1+T)\left(\|\theta\|_{\tilde{L}_{T}^{\infty} F_{p, q}^{s}}+\left\|\theta^{\prime}\right\|_{\tilde{L}_{T}^{\infty} F_{p, q}^{s}}\right)+\left\|\theta^{\prime}\right\|_{\tilde{L}_{T}^{1} \dot{F}_{p, q}^{s+1}}\right) .
\end{aligned}
$$

Since $\theta(t, x), \theta^{\prime}(t, x)$ satisfy (3.13), if $\epsilon$ and $T$ have been chosen small enough then it follows that $\delta \theta(t, x)=0$ on $[0, T] \times \mathbb{R}^{3}$. By a standard continuation argument, we can show that $\delta \theta(t, x)=0$ in $[0, \infty) \times \mathbb{R}^{3}$, i.e. $\theta(t, x)=\theta^{\prime}(t, x)$.

This completes the proof of Theorem 1.1.

\section{Appendix}

In this section, we provide the product estimate in the space-time Triebel-Lizorkin spaces which is used in the proof of Theorem 1.1. Chen and Zhang [18] established the estimate by the Littlewood-Paley trichotomy decomposition. Here we give a proof by the Bony paraproduct technique.

Lemma A.1 (Product estimate) Assume that $u, v \in \tilde{L}_{T}^{\infty}\left(F_{p, q}^{s}\left(\mathbb{R}^{N}\right)\right) \cap \tilde{L}_{T}^{1}\left(\dot{F}_{p, q}^{s+1}\left(\mathbb{R}^{N}\right)\right), s>\frac{N}{p}$, $1<p<\infty, 1 \leq q \leq \infty$. Then

$$
\|u v\|_{\tilde{L}_{T}^{1} \dot{F}_{p, q}^{s, 1}} \leq C\left(\|u\|_{\tilde{L}_{T}^{\infty} F_{p, q}^{s}}\|v\|_{\tilde{L}_{T}^{1} \dot{F}_{p, q}^{s+q}}+\|u\|_{\tilde{L}_{T}^{1} \dot{F}_{F, q}^{s+,}}\|v\|_{\tilde{L}_{T}^{\infty} F_{p, q}^{s}}\right)
$$

here $C$ is a constant only depending on s. 
Proof Using Bony's paraproduct decomposition and the property of quasi-orthogonality (2.6)-(2.7), $\forall j \in \mathbb{Z}$, we have

$$
\begin{aligned}
\dot{\Delta}_{j}(u v) & =\sum_{|k-j| \leq 4} \dot{\Delta}_{j}\left(\dot{S}_{k-1} u \dot{\Delta}_{k} v\right)+\sum_{|k-j| \leq 4} \dot{\Delta}_{j}\left(\dot{S}_{k-1} v \dot{\Delta}_{k} u\right)+\sum_{k \geq j-3} \sum_{|v| \leq 1} \dot{\Delta}_{j}\left(\dot{\Delta}_{k-v} u \dot{\Delta}_{k} v\right) \\
& =I+I I+I I I .
\end{aligned}
$$

Thus we have

$$
\begin{aligned}
\|u v\|_{\tilde{L}_{T}^{1} \dot{P}_{p, q}^{s+1}} & \leq\|\|\left(2^{j(s+1)}\|I\|_{L_{T}^{1}}\right)_{j \in \mathbb{Z}}\left\|_{l^{q}}\right\|_{L^{p}}+\cdots+\|\|\left(2^{j(s+1)}\|I I I\|_{L_{T}^{1}}\right)_{j \in \mathbb{Z}}\left\|_{l^{q}}\right\|_{L^{p}} \\
& =I^{\prime}+I I^{\prime}+I I I^{\prime} .
\end{aligned}
$$

We shall estimate the above three terms, respectively.

For the first term $I^{\prime}$ on the RHS of (A.3), by using the Minkowski inequality, the Young inequality, Lemma 2.1, and Lemma 2.2, we get

$$
\begin{aligned}
I^{\prime} & \leq\|\|\left(2^{j(s+1)} \sum_{|k-j| \leq 4}\left\|\mathcal{M}\left(\dot{S}_{k-1} u \dot{\Delta}_{k} v\right)\right\|_{L_{T}^{1}}\right)_{j \in \mathbb{Z}}\left\|_{l^{q}}\right\|_{L^{p}} \\
& \lesssim\|\|\left(2^{j(s+1)} \sum_{|k-j| \leq 4}\|u\|_{L_{T}^{\infty} L_{x}^{\infty}}\left\|\mathcal{M}\left(\dot{\Delta}_{k} v\right)\right\|_{L_{T}^{1}}\right)_{j \in \mathbb{Z}}\left\|_{l q}\right\|_{L^{p}} \\
& \lesssim\|u\|_{L_{T}^{\infty} L_{x}^{\infty}}\|\|\left(\sum_{|k-j| \leq 4} 2^{(j-k)(s+1)} \cdot 2^{k(s+1)}\left\|\mathcal{M}\left(\dot{\Delta}_{k} v\right)\right\|_{L_{T}^{1}}\right)_{j \in \mathbb{Z}}\left\|_{l q}\right\|_{L^{p}} \\
& \lesssim\|u\|_{L_{T}^{\infty} L_{x}^{\infty}}\|\|\left(2^{j(s+1)}\left\|\mathcal{M}\left(\dot{\Delta}_{j} v\right)\right\|_{L_{T}^{1}}\right)_{j \in \mathbb{Z}}\left\|_{l q}\right\|_{L^{p}} \\
& \lesssim\|u\|_{L_{x}^{\infty} L_{T}^{\infty}}\|\|\left(2^{j(s+1)}\left\|\dot{\Delta}_{j} v\right\|_{L_{T}^{1}}\right)_{j \in \mathbb{Z}}\left\|_{l^{q}}\right\|_{L^{p}} \\
& \lesssim\|u\|_{\tilde{L}_{T}^{\infty} F_{p, q}^{s}}\|v\|_{\tilde{L}_{T}^{1} \dot{F}_{p, q}^{s+1}}
\end{aligned}
$$

Similarly, for the second term $I I^{\prime}$ on the RHS of (A.3), we have

$$
I I^{\prime} \lesssim\|u\|_{\tilde{L}_{T}^{1} \dot{F}_{p, q}^{s+q}}\|v\|_{\tilde{L}_{T}^{\infty} F_{p, q}^{s}}
$$

Now, we turn to estimate the third term III' on the RHS of (A.3). From the Young inequality, Lemma 2.1, and Lemma 2.2, we have

$$
\begin{aligned}
I I I^{\prime} & \lesssim\|\|\left(2^{j^{(s+1)}} \sum_{k \geq j-3} \sum_{|v| \leq 1}\left\|\mathcal{M}\left(\dot{\Delta}_{k-v} u \dot{\Delta}_{k} v\right)\right\|_{L_{T}^{1}}\right)_{j \in \mathbb{Z}}\left\|_{l^{q}}\right\|_{L^{p}} \\
& \lesssim\|u\|_{L_{T}^{\infty} L_{x}^{\infty}}\|\|\left(2^{j(s+1)} \sum_{k \geq j-3}\left\|\mathcal{M}\left(\dot{\Delta}_{k} v\right)\right\|_{L_{T}^{1}}\right)_{j \in \mathbb{Z}}\|\|_{l q} \|_{L^{p}} \\
& \lesssim\|u\|_{L_{T}^{\infty} L_{x}^{\infty}}\|\|\left(2^{(j-k)(s+1)} \sum_{k \geq j-3} 2^{k(s+1)}\left\|\dot{\Delta}_{k} v\right\|_{L_{T}^{1}}\right)_{j \in \mathbb{Z}}\|\|_{l q} \|_{L^{p}} \\
& \lesssim\|u\|_{\tilde{L}_{T}^{\infty} F_{p, q}^{s}}\|v\|_{\tilde{L}_{T}^{1} F_{p, q}^{s+1}}
\end{aligned}
$$

Summing up (A.4)-(A.6), we obtain the product estimate (A.1). 
Next we give a decay estimate of the fractional heat semigroup $e^{-t v(-\Delta)^{\frac{\alpha}{2}}}$ on distributions with Fourier transforms supported in the annulus. The result is due to Chen and Zhang [18] in the 2D case and was used in a crucial way for the proof of Theorem 1.1. For convenience of the reader, we will give a short proof.

Lemma A.2 Let $\alpha \in(0,2], j \in \mathbb{Z}$. We have the following decay estimates:

$$
\begin{aligned}
& \left|e^{-t \nu \Lambda^{\alpha}} \dot{\Delta}_{j} f(x)\right| \leq C e^{-c t 2^{j \alpha}} \mathcal{M}\left(\dot{\Delta}_{j} f\right)(x), \quad \forall x \in \mathbb{R}^{3}, t>0, \\
& \left|e^{-t \nu \Lambda^{\alpha}} \nabla \cdot \dot{\Delta}_{j} f(x)\right| \leq C 2^{j} e^{-c t 2^{j \alpha}} \mathcal{M}\left(\dot{\Delta}_{j} f\right)(x), \quad \forall x \in \mathbb{R}^{3}, t>0,
\end{aligned}
$$

where $C$ and $c$ are positive constants independent of $t, j$.

Proof We consider a function $\phi \in \mathcal{D}\left(\mathbb{R}^{3} \backslash \mathcal{B}\left(0, \frac{1}{4}\right)\right)$, the value of which is identically 1 near the annulus $\mathcal{C}=\left\{\xi \in \mathbb{R}^{3}, \frac{3}{4} \leq|\xi| \leq \frac{8}{3}\right\}$. Denote

$$
e^{-t \nu \Lambda^{\alpha}} \dot{\Delta}_{j} f(x)=\mathcal{F}^{-1}\left(e^{-t \nu|\xi|^{\alpha}} \phi\left(2^{-j} \xi\right) \widehat{\dot{\Delta}_{j} f}(\xi)\right)(x)=: K_{j, t} * \dot{\Delta}_{j} f(x),
$$

where $K_{j, t}(x)=(2 \pi)^{-3} \int_{\mathbb{R}^{3}} e^{-t v|\xi|^{\alpha}} \phi\left(2^{-j} \xi\right) e^{i x \cdot \xi} d \xi$. Since

$$
K_{j, t}(x)=(2 \pi)^{-3} 2^{3 j} \int_{\mathbb{R}^{3}} e^{-t v 2^{j \alpha}|\xi|^{\alpha}} \phi(\xi) e^{i 2 x^{j} \cdot \xi} d \xi=: 2^{3 j} \widetilde{K}_{j, t}\left(2^{j} x\right)
$$

where $\widetilde{K}_{j, t}(x)=(2 \pi)^{-3} \int_{\mathbb{R}^{3}} e^{-t v 2^{j \alpha}|\xi|^{\alpha}} \phi(\xi) e^{i x \cdot \xi} d \xi$, we have

$$
\left|\widetilde{K}_{j, t}(x)\right| \lesssim \int_{\operatorname{supp} \phi} e^{-t \nu 2^{j \alpha}|\xi|^{\alpha}} d \xi \lesssim e^{-c t \nu 2^{j \alpha}}
$$

Next, we study the asymptotic behavior of $\widetilde{K}_{j, t}(x)$ for large $x$. Let $L(x, D)=\frac{x \cdot \nabla_{\xi}}{i|x|^{2}}$, then $L(x, D) e^{i x \cdot \xi}=e^{i x \cdot \xi}$. Consequently, $L^{*}=L^{*}(x, D)=-\frac{x \cdot \nabla_{\xi}}{i|x|^{2}}$, then for any $N_{*} \in \mathbb{N}$, we have

$$
\begin{aligned}
\widetilde{K}_{j, t}(x) & =(2 \pi)^{-3} \int_{\mathbb{R}^{3}} e^{-t v 2^{j \alpha}|\xi|^{\alpha}} \phi(\xi) L^{N_{*}}\left(e^{i x \cdot \xi}\right) d \xi \\
& =(2 \pi)^{-3} \int_{\mathbb{R}^{3}}\left(L^{*}\right)^{N_{*}}\left(\phi(\xi) e^{-t \nu 2^{j \alpha}|\xi|^{\alpha}}\right) e^{i x \cdot \xi} d \xi .
\end{aligned}
$$

The integrand in (A.11) can be majorized by

$$
|x|^{-N_{*}} \max \left(1,\left(t 2^{j \alpha}|\xi|^{\alpha-1}\right)^{N_{*}}, t 2^{j \alpha}|\xi|^{\alpha-N_{*}}\right) e^{-t \nu 2^{j \alpha}|\xi|^{\alpha}} \chi_{\operatorname{supp} \phi} .
$$

Thus, we have

$$
\begin{aligned}
\left|\widetilde{K}_{j, t}(x)\right| & \leq C_{N_{*}}|x|^{-N_{*}} e^{-c t v 2^{j \alpha}} \int_{\operatorname{supp} \phi}\left(1+t 2^{j \alpha}|\xi|^{\alpha}\right)^{N_{*}} e^{-\frac{-t v j^{j \alpha}}{2}|\xi|^{\alpha}} d \xi \\
& \leq C_{N}|x|^{-N_{*}} e^{-c t v j^{j \alpha}} .
\end{aligned}
$$

Combining (A.10) and (A.12), we deduce that

$$
\left|K_{j, t}(x)\right| \leq C_{N_{*}} 2^{3 j} e^{-c t v 2^{j \alpha}}\left(1+\left|2^{j} x\right|\right)^{-N_{*}} .
$$


Now, we turn to the proof of the inequality (A.7). For $N_{*}>3$, we have

$$
\begin{aligned}
\left|e^{-t v(-\Delta)^{\frac{\alpha}{2}}} \dot{\Delta}_{j} f(x)\right| & \left|\int_{\mathbb{R}^{3}} K_{j, t}(x-y) \dot{\Delta}_{j} f(y) d y\right| \\
\leq & C_{N_{*}} 2^{3 j} e^{-c t \nu 2^{j \alpha}} \int_{\mathbb{R}^{3}}\left(1+\left|2^{j}(x-y)\right|\right)^{-N_{*}}\left|\dot{\Delta}_{j} f(y)\right| d y \\
\leq & C_{N_{*}} 2^{3 j} e^{-c t \nu 2^{j \alpha}}\left[\int_{|x-y| \leq 2^{-j}}\left|\dot{\Delta}_{j} f(y)\right| d y\right. \\
& \left.+\sum_{l \geq 0} \int_{2^{-j+l} \leq|x-y| \leq 2^{-j+l+1}}\left(1+\left|2^{j}(x-y)\right|\right)^{-N_{*}}\left|\dot{\Delta}_{j} f(y)\right| d y\right] \\
\leq & C_{N_{*}} 2^{3 j} e^{-c t v 2^{j \alpha}}\left[\mathcal{M}\left(\dot{\Delta}_{j} f\right)(x) 2^{-3 j}+\sum_{l \geq 0} \int_{2^{-j+l} \leq|x-y| \leq 2^{-j+l+1}} 2^{-l N_{*}}\left|\dot{\Delta}_{j} f(y)\right| d y\right] \\
\leq & C_{N_{*}} 2^{3 j} e^{-c t \nu 2^{j \alpha}}\left[\mathcal{M}\left(\dot{\Delta}_{j} f\right)(x) 2^{-3 j}+\sum_{l \geq 0} 2^{-l N_{*}} 2^{-3 j+3 l} \mathcal{M}\left(\dot{\Delta}_{j} f\right)(x)\right] \\
\leq & C_{N_{*}} e^{-c t v 2^{j \alpha}} \mathcal{M}\left(\dot{\Delta}_{j} f\right)(x) .
\end{aligned}
$$

Thus we obtain the desired estimate (A.7).

Following the same procedure of estimate leading to (A.7), we can prove the estimate (A.8) similarly.

\section{Competing interests}

The authors declare that they have no competing interests.

\section{Authors' contributions}

All authors read and approved the final manuscript.

\section{Acknowledgements}

The authors are grateful to the anonymous referees for helpful comments and suggestions, which greatly improved the presentation of this paper. This work was supported by National Natural Science Foundation of China (Grant No. 11471129).

Received: 4 April 2016 Accepted: 9 June 2016 Published online: 17 June 2016

\section{References}

1. Castro, A, Córdoba, D, Gancedo, F, Orive, R: Incompressible flow in porous media with fractional diffusion. Nonlinearity 22, 1791-1815 (2009)

2. Guo, YT, Wang, M, Tang, YB: Higher regularity of global attractor for a damped Benjamin-Bona-Mahony equation on R. Appl. Anal. 94(9), 1766-1783 (2015)

3. Kang, JJ, Guo, YT, Tang, YB: Local well-posedness of generalized BBM equations with generalized damping on 1D torus. Bound. Value Probl. 2015, 227 (2015)

4. Yu, YH, Wu, X, Tang, YB: A magnetic regularity criterion for the $2 \mathrm{D}$ MHD equations with velocity dissipation. Bound. Value Probl. 2016, 113 (2016)

5. Córdoba, D, Gancedo, F, Orive, R: Analytical behavior of two-dimensional incompressible flow in porous media. J. Math. Phys. 48, 1-19 (2007)

6. Córdoba, D, Faraco, D, Gancedo, F: Lack of uniqueness for weak solutions of the incompressible porous media equation. Arch. Ration. Mech. Anal. 200, 725-746 (2011)

7. Bae, H, Granero-Belinchón, R: Global existence for some transport equations with nonlocal velocity. Adv. Math. 269, 197-219 (2015)

8. Hmidi, T, Keraani, S: Global solutions of the super-critical 2D Q-G equation in Besov spaces. Adv. Math. 214,618-638 (2007)

9. Xue, LT: On the well-posedness of incompressible flow in porous media with supercritical diffusion. Appl. Anal. 88(4), 547-561 (2009)

10. Kiselev, A, Nazarov, F, Volberg, A: Global well-posedness for the critical 2D dissipative quasi-geostrophic equation. Invent. Math. 167, 445-453 (2007) 
11. Yuan, B, Yuan, J: Global well-posedness of incompressible flow in porous media with critical diffusion in Besov spaces. J. Differ. Equ. 246, 4405-4422 (2009)

12. Yamazaki, K: Remarks on the method of modulus of continuity and the modified dissipative porous media equation. J. Differ. Equ. 250, 1909-1923 (2011)

13. Chae, D: On the well-posedness of the Euler equations in the Triebel-Lizorkin spaces. Commun. Pure Appl. Math. 55 654-678 (2002)

14. Chae, D: The quasi-geostrophic equation in the Triebel-Lizorkin spaces. Nonlinearity 16, 479-495 (2003)

15. Wang, $M$, Tang, YB: Long time dynamics of $2 D$ quasi-geostrophic equations with damping in $L^{p}$. J. Math. Anal. Appl. 412, 866-877 (2014)

16. Wang, $\mathrm{M}$, Tang, YB: On dimension of the global attractor for 2D quasi-geostrophic equations. Nonlinear Anal., Real World Appl. 14, 1887-1895 (2013)

17. Wang, $M$, Tang, YB: Attractors in $H^{2}$ and $L^{2 p-2}$ for reaction diffusion equations on unbounded domains. Commun. Pure Appl. Anal. 12(2), 1111-1121 (2013)

18. Chen, QL, Zhang, ZF: Global well-posedness of the 2D critical dissipative quasi-geostrophic equation in the Triebel-Lizorkin spaces. Nonlinear Anal. 67, 1715-1725 (2007)

19. Bahouri, H, Chemin, J, Danchin, R: Fourier Analysis and Nonlinear Partial Differential Equations. Springer, Berlin (2011)

20. Triebel, H: Theory of Function Spaces II. Monographs in Mathematics, vol. 84. Birkhäuser, Basel (1992)

21. Stein, EM: Harmonic Analysis: Real-Variable Methods, Orthogonality, and Oscillatory Integrals. Princeton Mathematical Series, vol. 43. Princeton University Press, Princeton (1993)

22. Stein, E: Singular Integrals and Differentiability Properties of Functions. Princeton University Press, Princeton (1970)

23. Frazier, M, Weiss, G, Torres, R: The boundedness of Caleron-Zygmund operators on the spaces $\dot{F}_{q}^{p, \alpha}$. Rev. Mat. Iberoam. 4, 41-72 (1988)

24. Córdoba, A, Córdoba, D: A maximum principle applied to quasi-geostrophic equations. Commun. Math. Phys. 249, 511-528 (2004)

\section{Submit your manuscript to a SpringerOpen ${ }^{\circ}$ journal and benefit from:}

- Convenient online submission

Rigorous peer review

- Immediate publication on acceptance

- Open access: articles freely available online

- High visibility within the field

- Retaining the copyright to your article 